\title{
Effectiveness of underlay myringoplasty in inactive mucosal chronic otitis media; a prospective study
}

\author{
Swapna U.P. ${ }^{1 *}$, Smitha B. ${ }^{2}$, Kumar K. S. ${ }^{3}$ \\ DOI: https://doi.org/10.17511/ijmrr.2020.i06.04
}

\footnotetext{
1* Swapna U.P., Associate Professor, Department of ENT, SUT Academy of Medical Sciences, Trivandrum, Kerala, India.

${ }^{2}$ Smitha B., Assistant Professor, Department of ENT, SUT Academy of Medical Sciences, Trivandrum, Kerala, India.

${ }^{3}$ Salil Kumar K., Professor, Department of ENT, SUT Academy of Medical Sciences, Trivandrum, Kerala, India.
}

Introduction: Patients with a mucosal type of chronic otitis media (COM) usually have recurrent ear discharge and conductive hearing loss. It is characterized by the central perforation of the tympanic membrane. Myringoplasty is an operation performed to repair tympanic membrane perforation when there is no ossicular damage. It helps to prevent ear discharge and improves hearing. Different techniques used for myringoplasty are underlay and overlay. Underlay technique is an effective procedure for tympanic membrane repair. Methods: 125 patients who underwent underlay myringoplasty over two years in a tertiary care center in Kerala were included in the study. Patients with actively discharging ears were excluded from the study. Results: The majority of the patients were in the age group of 20-29 years (49.6\%). There were 47 males (37.6\%) and 78 females $(62.4 \%)$. In 63 patients the surgery was done on the right side $(50.4 \%)$ and in 62 patients on the left side (49.6\%). Maximum preoperative hearing loss was in the range of $30-34 \mathrm{~dB}(39.2 \%) .100$ patients $(80 \%)$ showed successful graft uptake with the improvement of hearing in the range of 10 $15 \mathrm{~dB}$. Eight patients (6.4\%) showed small residual perforation with the improvement of hearing. In 17 patients $(13.6 \%)$ there was a complete rejection of graft with continuous ear discharge and no improvement in hearing. Conclusion: Underlay myringoplasty is an effective surgical technique for the repair of tympanic membrane perforations in terms of graft take up and improvement of hearing.

Keywords: Tympanic membrane, Chronic otitis media, Myringoplasty, Underlay technique

\section{Corresponding Author}

Swapna U.P., Associate Professor, Department of ENT, SUT Academy of Medical Sciences, Trivandrum, Kerala, India.

Email: swapnamanoj@gmail.com

\section{How to Cite this Article}

Swapna U P, Smitha B, Kumar K S. Effectiveness of underlay myringoplasty in inactive mucosal chronic otitis media; a prospective study. Int J Med Res Rev. 2020;8(6):398-403.

Available From

https://ijmrr.medresearch.in/index.php/ijmrr/article/ view/1229
To Browse

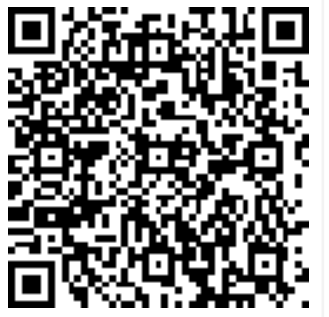

Manuscript Received 2020-11-26

Conflict of Interest No
Review Round 1 2020-12-06

Funding

Review Round 2
$2020-12-15$
Ethical Approval
Yes

Review Round 2

Yes
Review Round 3

Plagiarism X-checker $7 \%$
Accepted 2020-12-29

Note

(c) 2020 by Swapna U.P., Smitha B., Salil Kumar K. and Published by Siddharth Health Research and Social Welfare Society. This is an Open Access article licensed under a Creative Commons Attribution 4.0 International License https://creativecommons.org/licenses/by/4.0/ unported [CC BY 4.0]. 


\section{Introduction}

Chronic otitis media (COM) affects all age groups and both genders. COM equates the word Chronic suppurative otitis media (CSOM) used in some other research articles. The pathological subtypes of COM include inactive mucosal disease, active mucosal disease, inactive squamous epithelial disease, active squamous epithelial disease and healed otitis media [1]. In inactive mucosal COM, there is a permanent perforation of the tympanic membrane without ear discharge. Active mucosal COM is a disease of the middle ear cleft associated with inflammation and formation of mucopurulent discharge which comes out through the perforation in the pars tensa of the tympanic membrane. Inactive squamous epithelial COM is associated with retraction of the tympanic membrane, retraction pocket, or tympanic membrane atelectasis.

Active squamous epithelial COM is associated with cholesteatoma. In this type, the ear discharge is usually foul-smelling and maybe blood-stained. The foul-smelling discharge is due to osteitis. Cholesteatoma has bone eroding property and hence the chances of complications are more [1]. Other causes of perforation of the tympanic membrane are trauma to the ear or it may be due to iatrogenic causes [2]. The majority of the perforations heal spontaneously [3]. Persistent perforations in pars tensa can cause recurrent ear discharge and hearing loss. The ear discharge in these patients is profuse, mucopurulent, and nonfoul smelling. Both infratemporal and intracranial complications can develop in COM patients [4]. Common intratemporal complications are mastoiditis, labyrinthitis, and facial palsy. So, repair of the tympanic membrane is important in such patients to prevent discharge from the ear, improvement of hearing, and to reduce the incidence of complications.

Myringoplasty is an operation performed to repair a central perforation of the tympanic membrane. It is done in inactive mucosal chronic otitis media where there is a perforation of the tympanic membrane without ossicular damage. The techniques of myringoplasty include the underlay and overlay technique. In the underlay technique, the graft is placed medial to the tympanic membrane remnant. The most commonly used graft material is autologous temporalis fascia. Other graft materials include tragal perichondrium, tragal cartilage, ear lobule fat, or vein $[5,6]$.
Temporalis fascia graft is easy to harvest and the thickness is the same as that of the tympanic membrane. Various factors affect the outcome of surgery $[7,8]$. The diseased contralateral ear is a poor prognostic factor for graft uptake after myringoplasty [9]. Eustachian tube function is a good prognostic indicator of myringoplasty [10].

\section{Objectives of the study}

- To find the efficacy of underlay myringoplasty in inactive mucosal chronic otitis media in terms of graft take-up and improvement of hearing

- To analyze the incidence of complications after underlay myringoplasty

\section{Materials and Methods}

Setting: This study was conducted over 2 years in a tertiary care center in Kerala.

Type of study: Prospective study

Study population: Patients with inactive mucosal COM attending the outpatient ENT department

Sample size: 125 patients who underwent underlay myringoplasty and fulfilled the inclusion criteria

\section{Inclusion criteria}

- Patients with inactive mucosal chronic otitis media and mild to moderate conductive hearing loss.

\section{Exclusion criteria}

- Actively discharging ear

- Complicated chronic otitis media

- Chronic otitis media with cholesteatoma

- Patients with sensorineural or mixed hearing loss

- Elderly individuals and young children

- Patients undergoing myringoplasty by overlay technique

Data collection procedure: Informed consent was taken from all the patients. A detailed history including the onset of symptoms, duration, previous history of trauma or surgeries to the ear, any associated symptoms like tinnitus and vertigo were noted. History of diabetes and hypertension or any other systemic disease were recorded. Clinical examination including both general and otolaryngology examination was done for all patients. All these data were recorded in a proforma. 
Patients were selected for surgery only when the ear was dry for 6 weeks. Other prerequisites for myringoplasty were adequate cochlear reserve, absence of any focus of infection, and conductive hearing loss less than $40 \mathrm{~dB}$. Any predisposing factors like nasal allergy deviated nasal septum, sinusitis, rhinitis, tonsillitis, and pharyngitis were treated before surgery.17 patients with a history of nasal allergy were treated with antihistamines and intranasal steroids before surgery. 16 patients had a significant deviation of the nasal septum to the ipsilateral side and were corrected by surgery before myringoplasty. Two patients with recurrent tonsillitis were treated by tonsillectomy before surgery. In two patients ear discharge started after trauma to the ear. The otoscopic examination was done for all patients to assess the site of perforation, size of perforation, and status of middle ear mucosa. Twenty-three patients had inactive mucosal COM on the contralateral ear. In bilateral cases, the worst hearing ear was operated first Preoperative pure tone audiogram and X-ray mastoids lateral oblique view was taken for all patients. All the patients had conductive hearing loss of less than $40 \mathrm{~dB}$. The maximum preoperative hearing loss was $38.9 \mathrm{~dB}$. The surgery was done using an operating microscope. In 124 patients temporalis fascia was used as the graft material. In one patient with a small central perforation, ipsilateral ear lobule fat was used for myringoplasty. Antibiotics were given postoperatively. Regular follow-up was done during the postoperative period. The patients were followed up for a minimum period of 2 months. The patients were assessed for the subjective improvement of hearing, ear discharge, tinnitus, or vertigo. The otoscopic examination was done to assess the graft take up. A pure tone audiogram was taken for all patients in the postoperative period.

\section{Data analysis}

The data collected was recorded in a preformed proforma. Statistical analysis was done and the results were expressed as numbers and percentages in tables and as figures.

Ethical consideration: The study was approved by the institutional ethical committee.

\section{Results}

125 patients who underwent underlay myringoplasty were included in the study. The youngest was a 15-year-old female and the oldest, a 50-year-old lady.
The graft was taken in both the patients with significant improvement in hearing. The majority of the patients were in the age group of 20-29 years (49.6\%). The mean age was 28.1 years. There were 47 males (37.6\%) and 78 females (62.4\%). The gender difference did not influence graft take-up. The age and gender distribution are given in Table 1. In 63 patients the surgery was done on the right side $(50.4 \%)$ and in 62 patients on the left side $(49.6 \%)$ as shown in Figure 1 . Side of myringoplasty did not influence the outcome. In 64 patients myringoplasty was done by transcanal approach $(51.2 \%)$. These patients had a wide external auditory canal. In 58 patients with a narrow canal and more anterior perforations, a postural approach (46.4\%) was adopted. In 3 cases, the surgery was done by an endaural approach $(2.4 \%)$. The approaches for myringoplasty are shown in Table 2. These approaches were chosen as per the requirement of individual cases. Maximum preoperative hearing loss was in the range of 30-34 $\mathrm{dB}(39.2 \%)$. The range of pre-operative hearing loss is given in Figure 2.

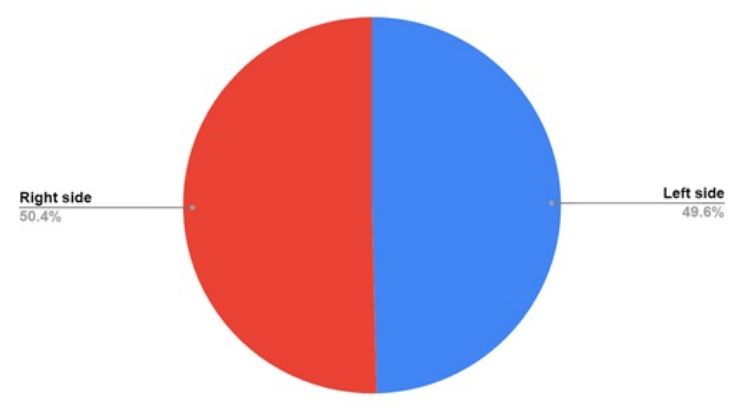

Fig-1: Side of myringoplasty

For the majority of the patients, the perforation was in the anteroinferior or posteroinferior quadrant. 34 patients had large central perforation (27.2\%), 74 patients $(59.2 \%)$ had medium-sized central perforation and 17 patients (13.6\%) had small central perforation. The size of perforation was found to have an association with the success of the surgery. Smaller perforations were found to have better results than larger ones. In the present study, 34 patients had large central perforation and the success rate was only $52.9 \%$. In these patients, there was a complete rejection of the graft in $29.4 \%$ and residual perforation in $17.6 \%$ cases.

Of the 125 patients, 100 patients (80\%) showed successful graft uptake with the improvement of hearing. Eight patients $(6.4 \%)$ showed a small residual perforation. 
These patients also showed an improvement in hearing. In 17 patients $(13.6 \%)$ there was a complete rejection of graft with continuous ear discharge and no improvement in hearing.

The most common cause of graft failure was infection during the postoperative period which started as an upper respiratory infection. One patient developed incision site granuloma which was treated by cauterization and repeated packing.

Table-1: Age and gender distribution of
patients.
\begin{tabular}{|l|l|l|l|}
\hline Age Range (years) & Male & Female & Total Number (Percentage) \\
\hline $10-19$ & 7 & 12 & $19(15.2 \%)$ \\
\hline $20-29$ & 23 & 39 & $62(49.6 \%)$ \\
\hline $30-39$ & 12 & 14 & $26(20.8 \%)$ \\
\hline $40-49$ & 5 & 12 & $17(13.6 \%)$ \\
\hline $50-59$ & 0 & 1 & $1(0.8 \%)$ \\
\hline Total & 47 & 78 & $125(100 \%)$ \\
\hline
\end{tabular}

Table-2. Approach to ear.

\begin{tabular}{|l|l|l|}
\hline \multicolumn{1}{|c|}{ Approach } & \multicolumn{1}{c|}{ Number } & \multicolumn{1}{c|}{ Percentage } \\
\hline Postaural & 58 & $46.4 \%$ \\
\hline Transcanal & 64 & $51.2 \%$ \\
\hline Endaural & 3 & $2.4 \%$ \\
\hline
\end{tabular}

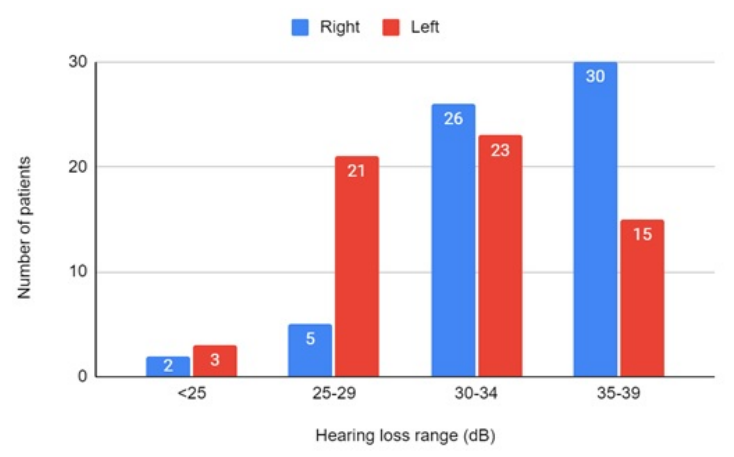

Fig-2: Preoperative hearing loss.

Of the 100 patients with accepted graft, the hearing improvement was in the range of 10-15 dB. None of the patients developed worsening of hearing, vertigo, sensorineural hearing loss, facial palsy, or tinnitus in the postoperative period.

\section{Discussion}

COM is an infection of the middle ear cleft. In inactive mucosal types of COM, there is perforation of the tympanic membrane without ear discharge. Myringoplasty is an effective surgical procedure to repair the tympanic membrane defect. It helps to improve the hearing and prevents further ear discharge.
The two common techniques of myringoplasty are underlay and overlay [2].

In a study by Alam $\mathrm{K}$ et $\mathrm{al}$, the graft take-up and hearing improvement were more in myringoplasty by postural approach than the other two approaches [11]. In the present study, different approaches were adopted as per the requirement of individual cases. Hence a definite opinion on approach and outcome parameters was not possible. A study by Aponte $\mathrm{RH}$ et al showed that eustachian tube function, postoperative care, size, and location of tympanic membrane perforation influences the hearing outcome after surgery. Large perforations showed less hearing improvement after tympanoplasty [7]. In another comparative study of underlay and overlay techniques by Mahesh et al, underlay myringoplasty was found to be more effective for large perforations and overlay technique for moderate-sized perforations [12] In a study by Kamath et al, a higher graft take-up rate was seen in large and medium perforations [13]. The correlation between the size of perforation and the outcome of myringoplasty is different in different studies. In the present study underlay, myringoplasty showed better results in small and medium-sized central perforations than larger ones. Jyothi et al conducted a comparative study of endoscopic versus microscopic myringoplasty in 120 patients divided into two groups of 60 each. The graft take-up was similar in both the groups, but the postoperative morbidity was less after endoscopic myringoplasty, The graft takeup in the microscopic group was 93.3\% [14]. The present study conducted myringoplasty using a microscope and the graft take-up was high.

There are many studies conducted to compare the results of underlay myringoplasty alone with underlay myringoplasty combined with cortical mastoidectomy. In one such study, the authors compared a group of patients treated by underlay myringoplasty alone with a group who were treated by underlay myringoplasty with cortical mastoidectomy in non-cholesteatomatous chronic otitis media [15]. There was no significant difference in the graft take-up in both patient groups ( $p$-value 0.7). Kamath et al in their comparative study showed that graft success rates were $80 \%$ in the group with myringoplasty alone and $88.33 \%$ in myringoplasty combined with cortical mastoidectomy, There was no statistically significant difference in the outcome of surgery in both the groups [13]. 
Another study to know the effectiveness of cortical mastoidectomy in myringoplasty by Silviu et al Showed cortical mastoidectomy has no additional benefit in myringoplasty[16], These studies show that myringoplasty alone gives good results in COM patients.

The present study results were comparable with the study conducted by Razaak MA et al, in 100 patients who underwent underlay myringoplasty and the success rate was $88 \%$ [17]. In their study, the majority of the patients were in the age group 3140 years $(38 \%)$, whereas, in the present study, the majority of the patients were in the age group 2029 years $(49.6 \%)$. The average audiological gain was $12.40 \mathrm{~dB}$ after myringoplasty in the study by Kamath et al [13]. In the present study, the hearing improvement after surgery was in the range of 10$15 \mathrm{~dB}$. Salmah MA et al, conducted a study on 67 patients to assess the outcome of myringoplasty reported a success rate of $94 \%$ at the end of one year [18].

A review of the literature showed that underlay myringoplasty is an effective surgical procedure to repair dry central perforations of the tympanic membrane. There is no significant difference in the outcome if it is combined with cortical mastoidectomy.

\section{Limitation}

The limitations of the present study were a small sample size and there was no long-term follow-up.

\section{Conclusions}

Underlay myringoplasty was an effective surgical procedure for the repair of the tympanic membrane in patients with inactive mucosal COM. $80 \%$ of patients showed successful graft take-up with the improvement of hearing. In $6.4 \%$ of cases, there was small residual perforation. These patients also showed an improvement in hearing. Small and medium-sized perforations showed better results than larger ones. Myringoplasty was a safe procedure and the complications were less. Only one patient developed incision site granuloma which was treated by cauterization.

\section{What does the study add to the existing knowledge?}

In mucosal chronic otitis media, there is ear discharge and hearing impairment.
There exists a social stigma related to recurrent ear discharge and impaired hearing. Underlay myringoplasty was a very effective surgical procedure to repair the tympanic membrane perforations in inactive mucosal COM and it reduces long-term morbidity.

\section{Author's contribution}

Dr. Swapna U.P. - Data collection, data analysis, and drafting the manuscript.

Dr. Smitha B.- Drafting the manuscript and editing.

Dr. Salil Kumar K. - Manuscript editing and manuscript review.

\section{Reference}

01. Michael G, Ray C, editors. Scott-Brown's otorhinolaryngology- head and neck surgery. 7th edition, London- CRC Press. 2008; 3.

doi: $\quad 10.1308 / 147870811 \times 598605 b \quad$ [Crossref]

02. Sergi B, Galli J, De Corso E, Parrilla C, Paludetti G. Overlay versus underlay myringoplastyreport of outcomes considering closure of perforation and hearing function. Acta Otorhinolaryngol Ital. 2011;31(6)366-371. [Crossref]

03. Gladstone HB, Jackler RK, Varav K. Tympanic membrane wound healing- An overview. Int Arch Otorhinolaryngol. 1995;28(5)913-932.

doi: $10.1016 /$ S0030-6665(20)30467-9 [Crossref]

04. Neeta S, Ashwin AJ, Praveer KB, Amrish KG. Complications of chronic suppurative otitis media and their management- A single institution 12 years' experience. Indian J Otolaryngol Head Neck Surg. 2015;67(4)353360 .

doi: $10.1007 / \mathrm{s} 12070-015-0836-5 \quad$ [Crossref]

05. Fernandes VLG, Goel HC, De Sousa E, De Gouveia Pinto NM. A comparative study of typeI underlay tympanoplasty with temporalis fascia graft alone and with conchal cartilage. Indian J Otolaryngol Head Neck Surg. 2019;71(2)13201326.

doi: $\quad 10.1007 / \mathrm{s} 12070-018-1397-1 \quad$ [Crossref] 
06. Liew L, Daudia A, Narula AA. Synchronous fat plug myringoplasty and tympanostomy tube removal in the management of refractory otorrhoea in younger patients. Int J Pediatr Otorhinolaryngol. 2002;66(3)291-296.

doi: $\quad 10.1016 / s 0165-5876(02) 00257-4 \quad$ [Crossref]

07. Aponte RH, Desentis VE, Vargas AA. Factors influencing hearing improvement of patients with chronic otitis media after tympanoplasty. Otorrinolaringología. 2007;52(1)22-28.

Available at: [Article] [Crossref]

08. Sarker M, Ahmed M, Patwary $K$, Islam $R$, Joarder $A$. Factors affecting surgical outcome of myringoplasty. Bangladesh J Otorhinolaryngol. 2011;17(2)82-87.

doi: $10.3329 /$ bjo.v17i2.8846 [Crossref]

09. Kripa D, Rakesh PS. Study of various prognostic factors affecting successful myringoplasty in a tertiary care centre. Int Arch Otorhinolaryngol. 2017;21(3)250-254.

doi: $10.1055 / \mathrm{s}-0036-1593818$ [Crossref]

10. Undavalli SB, Kulkarni NH, Bose S, Ananthaneni A. Tympanometric assessment of Eustachian tube function as a prognostic indicator in myringoplasty. Adv Arab Acad Audio-Vestibul J. 2017;4(1)14-18.

doi: 10.4103/aaaj.aaaj_4_17 [Crossref]

11. Alam K, Alam MM, Hossain MD, Karim MA, Hossain MA, Sarker MZ. Comparative study of different approaches of myringoplasty in chronic otitis media. Bangladesh J Otorhinolaryngol. 2020;22(1)21-25.

doi: 10.3329/bjo.v22i1.45074 [Crossref]

12. Mahesh SG, Vishwas KP, Pallavi P, Nithin PS. Myringoplasty- underlay versus overlay techniques - a comparative study. Int J Otorhinolaryngol head Neck Surg. 2018;4(2)381-386.

doi: $\quad$ 10.18203/issn.2454-5929.ijohns20180697

[Crossref]

13. Kamath MP, Sreedharan S, Rao AR, et al. Success of myringoplasty- our experience. Indian J Otolaryngol Head Neck Surg. 2013;65(4)358-362.

doi: $10.1007 / \mathrm{s} 12070-013-0651-9 \quad$ [Crossref]
14. Jyothi AC, Shrikrishna $\mathrm{BH}$, Kulkarni $\mathrm{NH}$, Kumar A. Endoscopic myringoplasty versus microscopic myringoplasty in tubotympanic CSOM- a comparative study of 120 cases. Indian J Otolaryngol Head Neck Surg. 2017;69(3)357362.

doi: 10.1007/s12070-017-1147-9 [Crossref]

15. Hazem MAT, Fadi MG, Tareq MA, Louay SES. Myringoplasty with and without cortical mastoidectomy in treatment of noncholesteatomatous chronic otitis media- a comparative study. Clin Med Insights Ear Nose Throat. 2014;7;19-23.

doi: $10.4137 /$ CMENT.S17980 [Crossref]

16. Silviu A, Franco T, and Maurizio A. Usefulness of Cortical Mastoidectomy in Myringoplasty. Otol Neurotol. 2012;33(4)604-609.

doi: $10.1097 / \mathrm{MAO} .0 \mathrm{~b} 013 \mathrm{e} 31825368 \mathrm{f} 2$ [Crossref]

17. Razzak MA, Murshed KM, Sobhan A, Hossain $M R$, Imtiaz $S N$. Outcome of myringoplasty in underlay technique. Bangladesh J Otorhinolaryngol. $2019 ; 24(2): 131-136$. doi: 10.3329/bjo.v24i2.44173 [Crossref]

18. Salmah MA, Wardah YAA, Talat AA, Ali SAQ. Myringoplasty outcome- Five-year experience at a tertiary teaching hospital. Saudi Med J. 2019;40(2)199-201.

doi: $\quad 10.15537 / \mathrm{smj} .2019 .2 .23896 \quad$ [Crossref]

